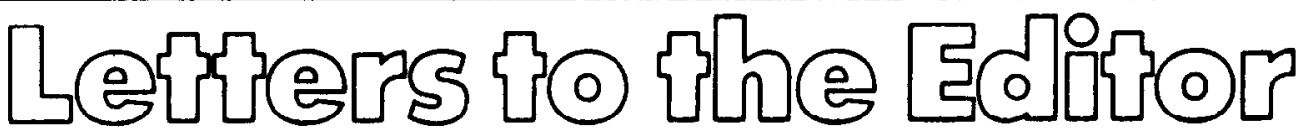

\section{LASER DOPPLER FLOW MEASUREMENTS WITH A SEMICONDUCTOR LASER}

Dear Sir,

In their report on laser Doppler flow measurements (J. Biomed. Eng. 1985, 7, 225) Boggett et al. compared, among other things, three different light sources for use in a laser Doppler device. The aim of that study was to distinguish between Doppler shifted and non-shifted backscattered light to obtain a flow dependent signal, which is not caused by intensity fluctuations due to number fluctuations, laser amplitude instabilities or other unwanted causes. We wish to comment on that paper.

We are surprised that only the Hughes He-Ne laser provided measurable Doppler shifted signals. In a previous report de Mul et al. ${ }^{1}$ have demonstrated that a laser Doppler device using a semiconductor laser can produce frequency spectra of a photocurrent in a detector, which is dependent on the Doppler shift of the backscattered light. These measurements were carried out on flowing particles in a tube.

To illustrate the in vivo performance of a laser Doppler instrument with a semiconductor laser as a light source, the spectra obtained from the skin blood flow of the third finger of a healthy volunteer are shown in Figure 1. For this purpose we used a laser Doppler device similar to that described previously by de Mul et al. ${ }^{1}$, with an RCA semiconductor (C86014E), instead of the Philips laser diode. The RCA laser diode produces

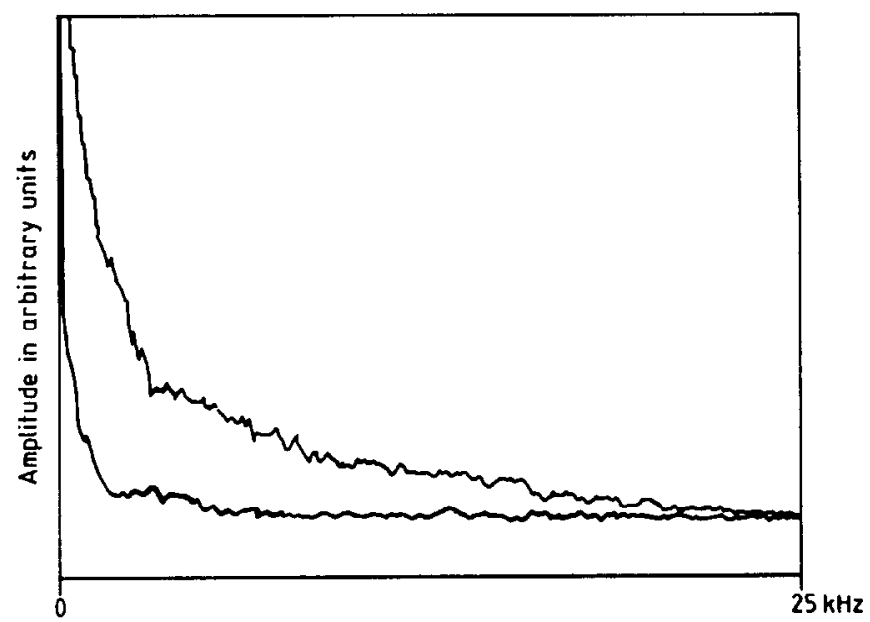

Figure 1 Amplitude spectra of the photocurrent, obtained with a semiconductor laser Doppler device. The upper spectrum represents undisturbed skin blood flow in the third finger. The lower spectrum is obtained during arterial occlusion. The spectra are recorded with a Hewlett-Packard 3561-A signal analyser after current-voltage conversion of the photocurrent
$5 \mathrm{~mW}$ of light power at a wavelength of $820 \mathrm{~nm}$ and a bandwidth of $0.02 \mathrm{~nm}$. We conclude that laser Doppler measurements of skin blood flow with a semiconductor laser as a light source are possible.

The reasons why Boggett et al. were not able to measure Doppler shifted signals using a semiconductor laser are not clear, partly because no further specifications of the laser are given. It may be possible that the coherence length was too short. However the fact that only the Hughes laser was able to produce measurable Doppler shifted signals indicates perhaps the presence of other disturbing factors, because other $\mathrm{He}-\mathrm{Ne}$ lasers used for Doppler flow measurements ${ }^{2-4}$ have proved to be reliable.

The first clinical results of flow measurements obtained by our semiconductor laser Doppler flow meter, which is already in use, will be published shortly.

Yours faithfully,

H.E. Suichies,
T.M. Smits,
J.G. Aarnoudse
Department of Obstetrics and Gynaecology
University Hospital Groningen, The Netherlands
H.W. Jentink,
F.F.M. de Mul,
J. Greve
Department of Applied Physics,
Twente University of Technology,
Enschede, The Netherlands

l de Mul, F.F.M., van Spijker, J., van der Plas, D., Greve, J., Aarnoudse, J.G. and Sinits, T.M. Mini laser-Doppler (blood) flow monitor with diode laser source and detection integrated in the probe. Appl. Optics 1984, 23, 2970-73

2 Nilsson, G.E., Tenland, T. and Oberg, P.A. A new instrument for continuous measurement of tissue blood flow by light beating spectroscopy. IEFF. Trans. Biomed. Fing. 1980, BME-27, 12-19

3 Smits, T.M. and Aarnoudse, J.G. Variability of fetal scalp blood flow during labour: continuous transcutaneous measurement by the laser Doppler technique. Br.J Obstet. Gynaecol. 1984, 91, 524-31

4 Wunderlich, R.W., Folger, R.L., Giddon, D.B. and Ware, B.R. Laser Doppler blood flow meter and optical plethysmograph. Rev. Sci. Instrum. 1980, 51, 1258-62 


\section{Dear Sir,}

Dr Aarnoudse et al. are correct in their comments that we were not able to measure Doppler shifted signals using a semiconductor laser and that a number of the He-Ne lasers we tested exhibited troublesome noise at low frequencies.

To take the question of the He-Ne lasers first. We found that of the limited number of lasers we tested all except the Hughes lasers were excessively noisy at frequencies in the range $0-20 \mathrm{kHz}$. Our aim in reporting this was to sound a warning to potential users and not to suggest that only one manufacturer produces lasers suitable for use in laser Doppler blood flow meters. Other workers have reported problems associated with low frequency laser-noise.

The semiconductor laser was the only one available to us during the period when we were making our measurements, and regretably it did not produce flow signals which could be attributed to Doppler shifts. We reported at the time that it was probable that the radiation generated by this laser, an RCA C86007E supplied to us in February 1982, was insufficiently coherent. Subsequent measurements have confirmed that the laser is indeed unsuitable for use in a laser Doppler skin blood flowmeter due to its short coherence lengths. We have recently renewed our interest in laser diodes as suitable radiation sources, having been made aware of the availability of relatively cheap semiconductor laser diodes with long coherence lengths. An example of such a laser is the Hitachi HL7801G which generates $5 \mathrm{~mW}$ at $790 \mathrm{~nm}$ and costs about

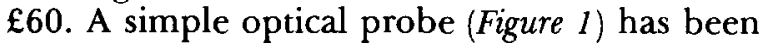
used by us for skin blood flow measurements and instrument calibration work.

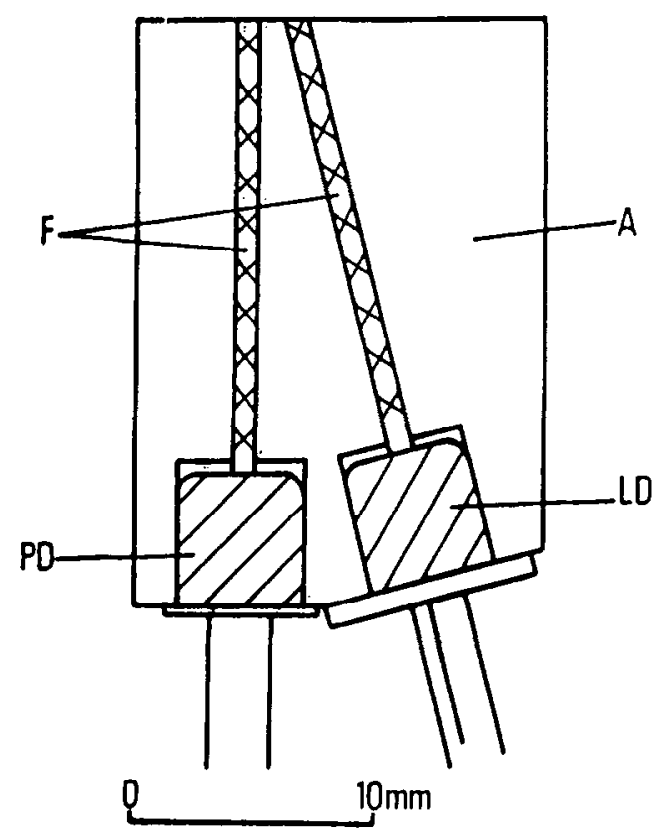

Figure 1 Diagram showing ronstruction of the laser diode optical probe. F, $1 \mathrm{~mm}$ plastic optical fibre; PD, photo diode; $\mathrm{LD}$, laser diode; $\mathrm{A}$, aluminium block

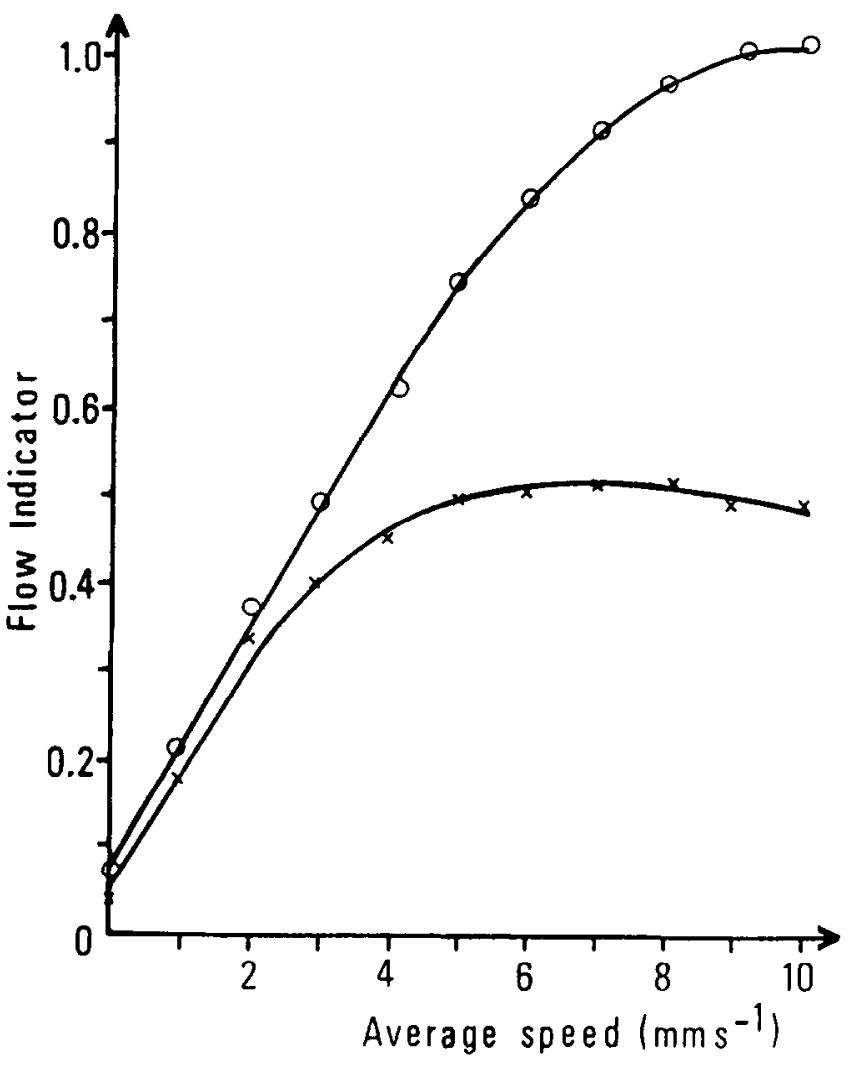

Figure 2 Flow index versus latex sphere average speeds for two instrument bandwidths. $0,10 \mathrm{~Hz}-20 \mathrm{kHz} ; \mathrm{X}, 10 \mathrm{~Hz}-$ $10 \mathrm{kHz}$

Results similar to those reported by de $\mathrm{Mul}^{1}$ are shown in Figure 2 where the flow indicator has been calculated from the integral of the first moment of the power spectral density of the photodiode current $^{2,3}$.

Yours faithfully,
D. Boggett, A. Obeid, J. Blond

Department of Geology and Physical Sciences, Oxford Polytechnic, Oxford, UK

P. Rolfe,

Bio-Engineering Centre University of Oxford John Radcliffe Hospital. Oxford, UK 